Annals of Mathematics Studies

Number 202 



\section{Arithmetic and Geometry}

Ten Years in Alpbach

EDITED BY

Gisbert Wüstholz and Clemens Fuchs

PRINCETON UNIVERSITY PRESS

PRINCETON AND OXFORD 
Copyright (C) 2019 by Princeton University Press

Requests for permission to reproduce material from this work should be sent to permissions@press.princeton.edu

Published by Princeton University Press

41 William Street, Princeton, New Jersey 08540

6 Oxford Street, Woodstock, Oxfordshire OX20 1TR

press.princeton.edu

All Rights Reserved

Library of Congress Control Number:2019943220

ISBN 978-0-691-19378-6

ISBN (pbk.) 978-0-691-19377-9

British Library Cataloging-in-Publication Data is available

Editorial: Vickie Kearn, Susannah Shoemaker, and Lauren Bucca Production Editorial: Nathan Carr

Text Design: Leslie Flis

Jacket/Cover Design: Leslie Flis

Production: Jacquie Poirier

Publicity: Matthew Taylor and Katie Lewis

This book has been composed in $\mathrm{AT}_{\mathrm{E}} \mathrm{X}$

Printed on acid-free paper. $\infty$

Printed in the United States of America

$\begin{array}{llllllllll}10 & 9 & 8 & 7 & 6 & 5 & 4 & 3 & 2 & 1\end{array}$ 